\title{
Theoretical Kinetic Studies of the Hydrodehalogenation Reaction of CBrClF2 (Halon-1211)
}

\author{
Alessandra F. Albernaz ${ }^{1 *}$ (PQ) and Patrícia R. P. Barreto² (PQ). alessandra@fis.unb.br \\ ${ }^{1}$ Instituto de Física, Universidade de Brasíllia, Brasília, DF, Brazil \\ ${ }^{2}$ Instituto Nacional de Pesquisas Espaciais, São José dos Campos, SP, Brazil \\ Keywords: Halon-1211, $\mathrm{CClBrF}_{2}$, Hydrodehalogenation, Variational Transition State Theory
}

\section{Introduction}

Halons (brominated fluorocarbons/chlorofluorocarbons-CFCs) represent a group of chemicals exhibiting desirable properties for a broad range of industrial and consumer applications, including refrigeration, fire suppression and manufacturing of polymeric foams. Unfortunately, these chemicals are potent ozone depleting substances (ODSs) and synthetic green house gases (GHGs). The wide use of these chemicals has caused severe damage to the ozone layer and contributed significantly to the global warming. In order to hasten the recovery of the ozone layer, international legislation, the Montreal Protocol, mandated the phase out of production of ODSs by all countries according to a specified timetable, which, in turn, has sparked worldwide research interest on the treatment of stockpiled CFC and Halon [1]. Model development of thermal degradation processes of halogenated compounds in flames requires detailed knowledge of the mechanisms governing such reactions as well as of the pertinent kinetic and thermodynamic data. This information is particularly invaluable in the understanding of the elementary mechanisms involved in the incineration of hazardous industrial wastes, which could aid scientists in the development of more cost effective and efficient incineration methods that will lead to more favorable environmental impacts. This is particularly true in the case of halogen abstraction from CBrClF2 (halon-1211) by hydrogen atom under combustion conditions

$$
\begin{aligned}
& \mathrm{H}+\mathrm{CBrClF} 2 \rightarrow \mathrm{HF}+\mathrm{CBrClF} \\
& \mathrm{H}+\mathrm{CBrClF} 2 \rightarrow \mathrm{HCl}+\mathrm{CBrF} 2 \\
& \mathrm{H}+\mathrm{CBrClF} 2 \rightarrow \mathrm{HBr}+\mathrm{CClF} 2
\end{aligned}
$$

which has been known to play an important role in incineration processes producing toxic materials. Quantum chemical calculations in conjunction with transition state theory (TST) can help in reliably assessing the relative importance of the elementary reactions of combustion processes such as the one described in R1-R3 without the need for empirical and complicated extrapolation procedures based on low-temperature data.

\section{Methodology}

An extensive quantum chemical study of the potential energy surface for the different pathways of the reactions of $\mathrm{H}+\mathrm{CBrClF} 2$ has been studied. We investigated the channels with detailed mapping of the potential energy surface, used CBS-QB3 Method to all species (reactants, transition states and products) involved in the reaction. To optimize the geometries and determine the vibrational frequencies of the reactants, transition states and products of the $\mathrm{H}+\mathrm{CBrClF} 2$ reaction it is used the B3LYP functional with the $6-311 \mathrm{G}(2 \mathrm{~d}, \mathrm{~d}, \mathrm{p})$ basis set. The vibrational frequencies with Intrinsic Reaction Coordinate (IRC) calculations were performed to confirm the nature of the global conformation minimum (no imaginary frequency) and transition state or saddle 
point (only one imaginary frequency). The canonical variational transition state theory (VTST/CVT) is employed to calculate the rate constants by APUAMA program [2].

\section{Results and Discussion}

The optimized geometric parameters of the reactant $(\mathrm{H}, \mathrm{CBrClF} 2)$, products ( $\mathrm{HF}, \mathrm{HCl}, \mathrm{HBr}, \mathrm{CBrClF}, \mathrm{CBrF} 2$ and $\mathrm{CClF} 2$ ) and transition states (Ts1 HF, Ts2 HCl, Ts $3 \mathrm{HBr})$ calculated at the B3LYP/6-311G(2d,d,p) level along are presented in Fig. 1. The Figure 2 shows the potential energy surface, with ZPE correction.
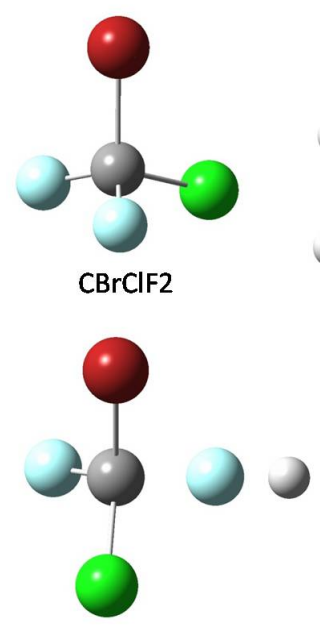

Ts1HF
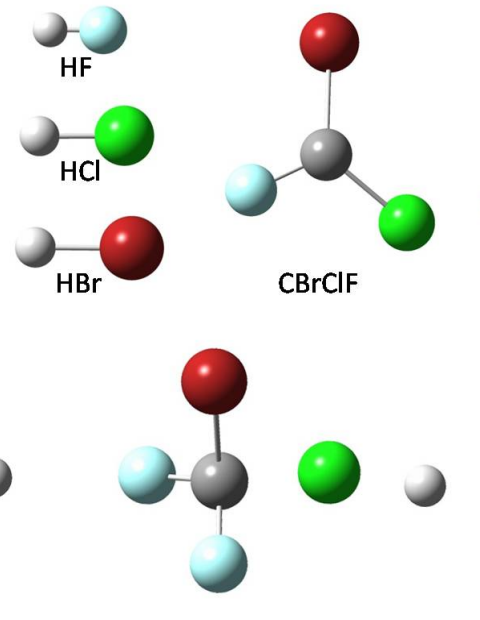

$\mathrm{Ts} 2 \mathrm{HCl}$

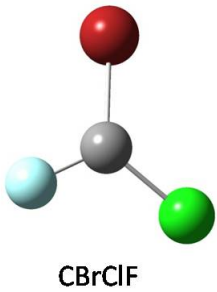

CBrCIF
CBrF2

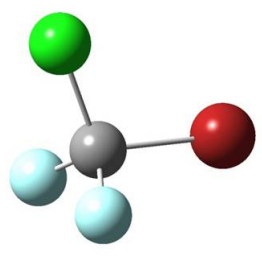

$\mathrm{Ts} 3 \mathrm{HBr}$

Figure 1. Optimized geometries of the reactant, products and transition states at the B3LYP/6-311G(2d,d,p) level.

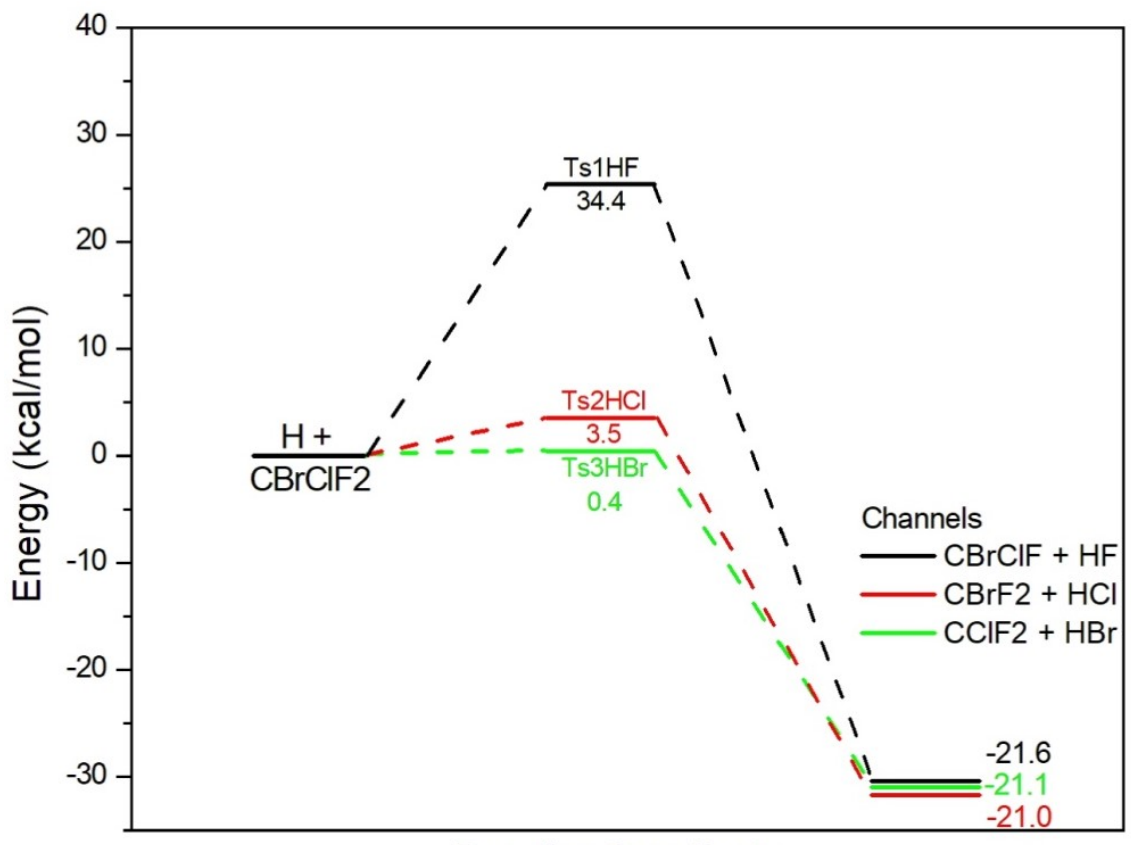

Reaction Coordinate

Figure 2. Schematic potential energy diagram for the $\mathrm{H}+\mathrm{CBrClF} 2$ reaction calculated using CBS-QB3 level, included ZPE.

\section{References}

[1] UNEP (2000) http://www.unep.org/ozone/pdf/Montreal-Protocol2000.pdf

[2] H. O. Euclides, P. R. P. Barreto, J. Mol. Model. (2017) 23: 176. 